"This is the peer reviewed version of the following article: MacDonald K, Malla A, Joober R, Shah JL, Goldberg K, Abadi S, Doyle M, lyer SN. Description, evaluation and scale-up potential of a model for rapid access to early intervention for psychosis. Early Interv Psychiatry. 2018 Dec;12(6):1222-1228., which has been published in final form at doi: 10.1111/eip.12564. This article may be used for non-commercial purposes in accordance with Wiley Terms and Conditions for Use of Self-Archived Versions."

\title{
A model for rapid access to early intervention for psychosis: Description, evaluation and scale-up potential
}

\author{
Kathleen MacDonald ${ }^{1,2,3}$, Ashok Malla ${ }^{1,2,3}$, Ridha Joober ${ }^{1,2,3}$, Jai L. Shah ${ }^{1,2,3}$, Karen Goldberg ${ }^{4}$, \\ Sherezad Abadi ${ }^{4}$, Madeline Doyle ${ }^{5}$, Srividya N. Iyer ${ }^{1,2,3}$ \\ ${ }^{1}$ Department of Psychiatry, McGill University, Montreal, Canada \\ ${ }^{2}$ Prevention and Early Intervention Program for Psychosis (PEPP), Douglas Mental Health \\ University Institute, Montreal, Canada \\ ${ }^{3}$ ACCESS Open Minds (Pan-Canadian youth mental health services research network), Douglas \\ Mental Health University Institute, Montreal, Canada \\ ${ }^{4}$ Douglas Mental Health University Institute, Montreal, Canada \\ ${ }^{5}$ MBA Candidate, Concordia University, Montreal, Canada
}

Corresponding Author: Srividya N. Iyer, Ph.D., Douglas Mental Health University Institute, ACCESS Pavilion, 6875 Boulevard La Salle, Montreal, Quebec H4H 1R3, Canada. srividya.iver@douglas.mcgill.ca

\section{Acknowledgments}

Iyer is supported by a salary award from the Canadian Institutes of Health Research (CIHR). Iyer, Joober and Shah have also been supported by Clinician-Scientist salary awards from the Fonds de recherche du Québec-Santé. Malla is supported by the Canada Research Chair program. This work is part of a larger program of research in early intervention and youth mental health supported by a CIHR Foundation Scheme grant. We appreciate the support by the PEPP clinical and research staff over the years, particularly those who have played the role of the PEPP intake clinician including Lucas Coulson, Thomas Holmes, Karen Goldberg, Melissa Palander and Simon Larivière. 
1 Abstract

2 Aim: This paper aims to describe the entry protocol of the Prevention and Early Intervention for

3 Psychosis Program (PEPP)-Montreal, an early intervention program for psychosis. The protocol is

4 designed to fulfill a key objective of the early intervention movement - reducing delays to accessing

5 high-quality care. The paper also aims to describe how this rapid entry protocol can be deployed in other

6 services interested in reducing delays in initiating treatment.

7 Methods: PEPP provides rapid, easy access to quality care by placing a single, well-trained professional,

8 the intake clinician, at the point of entry. Anyone can refer a youth directly and without formalities to the

9 intake clinician who responds promptly and sensitively to all help-seeking, whether by a youth, a family

10 member, a school counsellor or anyone acting on behalf of a youth in need. To promote accessibility,

11 PEPP guarantees an initial assessment within 72 hours; maintains relationships with referral sources; and

12 conducts awareness-enhancing outreach activities.

13 Results: Since 2003, PEPP has received 1,750 referrals, which have all been responded to within 72

14 hours. Families have been involved in the intake process in $60 \%$ of the cases and hospitalization may

15 have been averted in over half of the referrals originating from emergency-room services. Another

16 indicator of success is the very low turnover in the intake clinician's position. Overall, the PEPP model has

17 succeeded in providing rapid, engaging, easy and youth-friendly access to high-quality care.

18 Conclusion: The success of this protocol at PEPP has inspired the entry protocols at other first-episode

19 psychosis services. Its ability to provide rapid, engaging access to high-quality services may allow this

20 protocol to become a model for other early intervention services for psychosis and other mental illnesses.

\section{Keywords}

22 health services accessibility, early intervention, first episode psychosis, intake, youth

\section{Introduction}

24 Early intervention for psychosis comprises two conceptual components —reduction of treatment delays 25 and enhanced, phase-specific treatment (e.g., low-dose antipsychotic therapy, case management, family

26 interventions, etc. (Norman and Malla, 2001, Iyer, 2014) Many early intervention services prioritize

27 enhanced care, but not all specifically aim to reduce treatment delays, including delays after the initial 
28 referral (White et al. 2015). This is true despite the growing recognition that the presence of high-quality 29 services alone cannot ensure timely treatment: reducing treatment delays may also require focused, well30 conceived interventions (Lloyd-Evans et al., 2011).

31 The impetus to reduce treatment delay (measured as duration of untreated psychosis, or DUP) is rooted 32 in the notion that prolonged DUP is detrimental on many fronts (Marshall et al., 2005, Birchwood, 1999). 33 Two themes prevail in the literature on reducing treatment delays in first-episode psychosis. The first

34 focuses on formally-developed, systematic early case identification initiatives (Srihari et al., 2014, 35 McGorry et al., 1996, Malla et al., 2014). The second concerns pathways to care (Norman et al., 2004) 36 before reaching early intervention services, as research shows that multiple steps are often necessary to 37 reach early intervention services, often through circuitous, difficult, disengaging and traumatic pathways 38 (Singh and Grange, 2006).

39 DUP includes delays before help is first sought (help-seeking delay); delays between help-seeking and 40 referral to an early intervention service (referral delay); and delays between being referred and receiving 41 care (engagement delay) (Bechard-Evans et al., 2007). Reducing help-seeking and referral delays, and 42 simplifying referral routes to early intervention services is certainly important. However, it is equally 43 important to ensure that once referred (or self-referred) to early intervention services, people have rapid, 44 direct and engaging access to an initial evaluation and care. As the International Early Psychosis 45 Association Writing Group's standards outline, "Mental health services should provide user-friendly easy 46 access to assessment and treatment... Assessment should be timely, depending on urgency, and 47 flexible in terms of location." (IEPA, 2005)

48 Despite this consensus, little is known about whether and how early intervention services ensure rapid, 49 engaging access. Approaches vary widely and even early intervention services can suffer from poor 50 accessibility. A survey found wide variations across Canada in the mechanisms of entry into early 51 intervention services and delays between referral and care (Nolin et al., 2016).

52 It is also unknown whether entry to early intervention services is easier or faster than to regular care. If 53 pathways to El services are indeed simpler and post-referral delays shorter, the mechanisms in place to 54 achieve this have rarely been described in the literature. This is an important knowledge gap because the 
55 way a service responds to help-seeking may have implications for the future service engagement of 56 youths and carers (Andrade et al., 2014). Knowing how well services respond to referrals can constitute a

57 continuous, inexpensive and scalable means of improving case identification. A prompt, friendly and

58 sensitive response to referrals and consistent feedback regarding outcome of the referral to the referral

59 source can prompt referral sources to make more and timelier referrals.

60 This paper describes an innovative easy-entry, rapid-response assessment system at a Canadian first-

61 episode psychosis program and perspectives acquired over its 14-year history. We will also describe the

62 opportunities and challenges encountered in scaling this model up to emerging broad-spectrum youth

63 mental health services nationwide.

\section{$64 \quad$ Methods}

\section{Setting}

66 The Prevention and Early Intervention Program for Psychosis (PEPP) is a clinical-research unit at a

67 Montreal psychiatric hospital affiliated to McGill University. The program, established in 2003, was

68 modelled on one in London, Ontario. PEPP serves young persons with first-episode psychosis from a

69 specific catchment area with a population of 400,000 . Its services are free and covered by public

70 healthcare.

71 Inclusion criteria for follow-up at PEPP are: age 14 to 35 years old; DSM-IV diagnosis of non-affective or

72 affective psychotic disorder. Exclusion criteria are: organic causes (e.g. epilepsy), IQ of less than 70,

73 previous exposure to antipsychotic medication of greater than one-month duration. and substance-

74 induced psychosis (a comorbid diagnosis of substance abuse or dependence is not an exclusion

75 criterion).

76 The inclusion/exclusion criteria for the PEPP program were established given its mandate as a clinical-

77 research program providing services within a given catchment area. In order to ensure that the program is

78 providing services to young people presenting with a previously untreated and unresolved first episode of 79 psychosis, the 30-day exposure to antipsychotic medication exclusion criterion is used. 
80 Following international guidelines for specialized early intervention services for psychosis (IEPA, 2005),

81 PEPP offers assertive outreach and follow-up for two years through case management and

82 pharmacological and psychosocial interventions (Iyer et al., 2015b). Treatment focuses not only on

83 symptom remission but also on service engagement and functional and subjective recovery. PEPP's

84 clinical team currently includes one full-time clinical coordinator; one full-time intake clinician; 5

85 psychiatrists (who between them offer full-time coverage); 7.5 full-time case managers from various

86 backgrounds (social work, nursing, occupational therapy and allied disciplines); part-time staff including a

87 nutritionist, an employment specialist, psychologists; and student interns offering a range of treatments

88 such as cognitive-behavioural therapy, drama therapy, art therapy, and various group interventions.

89 PEPP's treatment protocol is described in greater detail elsewhere (lyer 2015b).

\section{Key principles guiding access to PEPP}

91 The following are core features of PEPP's entry and assessment model that, together, ensure direct,

92 rapid and engaging access to its services.

\section{Open and direct referral system}

94 To limit barriers to access, PEPP offers an open and direct referral system. Referrals can come from any

95 source (youths themselves or their families, teachers, health professionals, emergency departments, or

96 others acting on their behalf, etc.). No forms or official procedures are required (e.g., a referral from a

97 general practitioner is not a prerequisite). Initial contact can be made in person or by phone or email.

\section{$98 \quad$ Guaranteed quick-response protocol}

99 All referral sources are directly contacted by PEPP and an initial evaluation is offered within 72 hours.

100 Though PEPP usually responds within 24 hours, a 72-hour limit is set to accommodate weekends. The

101 initial contact is most often over the phone, and used to facilitate engagement early on and to go over

102 basic inclusion/exclusion criteria (e.g., age).

103 Intake clinician: A well-publicized, engaging, single point of first contact 
104 The key to PEPP's guarantee of rapid, engaging access is a trained non-physician mental health

105 professional called the intake clinician. Serving as a single point of initial contact, this clinician is directly

106 accessible and responds promptly to referrals. This clinician's time is dedicated entirely to responding to

107 help-seekers and conducting initial evaluations.

108 As the first point of contact, the intake clinician fosters service engagement early on by adopting a youth109 friendly, engaging attitude; offering flexibility and choice in the location and timing of assessment; and

110 focusing the intake interview on clients' personally salient goals (e.g., resuming school/work). Depending 111 on clients' or carers' preferences, the intake clinician meets them at PEPP, their home or school or a 112 community location like a café.

113 Many youths with first-episode psychosis live with or are close to their families (Szmukler and Bloch, 114 1997)) who often initiate help-seeking (Anderson et al., 2013, Boydell et al., 2006). Because family 115 involvement is known to improve long-term service engagement, medication adherence and outcomes 116 (Coldham et al., 2002, Doyle et al., 2014) the intake clinician engages families at the outset by involving 117 them in the initial assessment (except if clients forbid it).

118 Some patients are not invited for a face-to-face initial evaluation if it is ascertained in the initial phone 119 contact that PEPP services may not be appropriate, considering PEPP's inclusion/exclusion criteria (e.g., 120 client is older than 35). For all others, the intake clinician conducts an initial, face-to-face evaluation 121 (using a semi-structured intake interview guided by the client's needs and pace) to establish if PEPP 122 services are appropriate. When necessary, she consults with a psychiatrist, to whom she has immediate 123 access, to confirm the nature of the presenting problem, assess risk and promptly initiate any appropriate 124 psychopharmacological interventions and medical evaluations (EEG, MRI, etc.). Clients can receive a 125 psychiatrist's evaluation outside PEPP, at a location of their choosing.

126 Sometimes, the intake clinician initially works only with the family member or significant adult (e.g., school 127 counselor) who first contacted PEPP. She supports this carer and coaches them on how to propose help128 seeking to their young family member. The intake clinician provides family members basic 129 psychoeducation; offers them support and advice for dealing with crises; and links them to pertinent 
130 community supports and services. Family members may also be linked to PEPP's family peer support 131 group directly during the intake process.

132 The intake clinician orients clients and their families/carers to what can be expected from treatment. She 133 uses the first contact as an opportunity to establish clients' needs and goals, and discuss how PEPP can 134 help them achieve their recovery aspirations. This prioritization of personally salient goals (rather than 135 symptoms) often proves significant in securing the engagement of youths who may not otherwise be open 136 to receiving services for psychosis.

137 Whether or not a youth is accepted into PEPP, the intake clinician often contacts referrers with an update. 138 This fortifies links with referral sources and encourages more and more appropriate referrals in the future. 139 When PEPP services are inappropriate, the intake clinician often recommends and/or makes referrals to 140 apt services, helping youths and their families navigate a complex healthcare system. Though they are 141 sometimes beyond the purview of a typical intake process, these additional steps help connect youths to 142 appropriate services quickly. If outside treatment deemed urgently necessary (e.g. for a severe manic 143 episode without psychotic symptoms) is not immediately available, the PEPP team initiates treatment and 144 continues following clients until their transfer to more appropriate services.

145 Thus, by fulfilling diverse roles and functions, the intake clinician ensures that young people and their 146 families have early and engaging access to treatment, even if it is not at PEPP.

\section{Strong linkages through early identification and outreach}

148 As a catchment-based program, PEPP gets referrals from several fixed sources in the community.

149 (Rickwood et al., 2007) For early case identification (Friis et al., 2005), it is important that these frontline 150 entities recognize the signs and symptoms of psychosis and know how to refer people to appropriate 151 services. Close connections with these referral sources have helped reduce the DUP and make pathways 152 to care more direct and less traumatic. To that end, PEPP's ongoing early identification and outreach 153 activities include visits to general practitioners and schools; distributing pamphlets; academic detailing on 154 signs of psychosis using videos ; and highlighting PEPP's open referral system. Many of these materials, 155 including the video advertisements, were developed for a formal early identification study conducted in 
1562006 focused on the education of all potential referral sources (Malla et al., 2014). These in-person

157 presentations and booster sessions were facilitated by PEPP staff, particularly the intake clinician. Most

158 of PEPP's early identification and outreach activities involve the intake clinician. This helps potential

159 referral sources connect personally with the clinician who will handle their referrals.

\section{Results}

161 Below are key results from the implementation of PEPP's entry and assessment model.

\section{Open and direct referral system}

163 From January 2003 to May 2016, PEPP had over 1,750 referrals. By volume, the largest referral sources 164 were emergency services, family/self-referrals and inpatient units. Referral sources have become 165 increasingly diverse over time (Figure 1).

\section{Guaranteed, quick-response protocol}

$167100 \%$ of referrals to PEPP since 2003 were responded to within the 72 -hour benchmark. The median 168 time from referral to official entry into PEPP following initial evaluation was 16 days Official entry into the 169 PEPP program occurs after the initial evaluation by the intake clinician and a formal psychiatric 170 assessment (see Figure 2).

\section{Intake clinician}

172 Qualifications, training and support

173 Continuously since 2003, PEPP has operated with a single intake clinician whose background has been 174 in social work, nursing, psychology or allied professions. There has been little turnover in this role, with 175 the current clinician being with PEPP since 2013 and his predecessor holding the post for nine years. 176 This stability has allowed the intake clinician to develop and sustain relationships with referral sources in 177 the community. It reflects the support and satisfaction intake clinicians enjoy and suggests that the model 178 may be practically and feasibly implemented in other early intervention settings.

179 Building and sustaining the intake clinician's capacities through initial training and continuous supervision 180 has been instrumental to the model's success. Their training has revolved around the precepts and 
181 philosophy of early intervention, the signs and symptoms of psychosis, psychoeducation, risk assessment

182 and clinical measures/tools. Trainees shadow trained intake clinicians and clinical staff and are

183 introduced to PEPP's family support group coordinator and key health and social service partners. The

184 entire PEPP team meets at weekly rounds to present and discuss intake reports and generate group

185 feedback on complex, ambiguous or difficult presentations. More systematic reviews of response to

186 referrals in terms of delays, approach, emphasis on engagement, etc. occur every quarter.

187 PEPP's intake clinicians have received approximately 170 referrals annually. Around $60 \%$ of these 188 referrals have met PEPP's inclusion criteria. Of those excluded, 44\% did not have psychosis; $4 \%$ did not 189 meet the age criterion; $13 \%$ had taken antipsychotic medication for over one month; $34 \%$ did not live in 190 the catchment; and 5\% were not accepted for other reasons (e.g., symptoms explained by medical 191 conditions, loss of contact, etc.) The proportion of referrals not meeting inclusion criteria has remained 192 unchanged since 2003. However, there have been peaks in referrals associated with press releases or 193 other media attention, with a majority of these involving referrals not eventually appropriate for PEPP.

195 In a significant number of cases, family members or carers have been involved in the intake process, 196 usually being present in person. Since 2012, when family involvement began being systematically 197 recorded, families have been involved in over $60 \%$ of initial assessments. In $7 \%$ of cases, clients explicitly 198 forbade family involvement. In $33 \%$ of cases, extenuating circumstances (e.g. clients being international 199 students, refugees, etc.) precluded family involvement.

\section{Early identification and outreach}

201 Because most referrals to PEPP come from its host hospital's emergency department, the intake clinician 202 has fostered a close relationship with its staff. When presented with a youth who may meet PEPP criteria, 203 emergency staff contact the intake clinician who either initiates an immediate evaluation, or engages the 204 client and schedules an in-person meeting at PEPP as soon as possible. This may have drastically 205 minimized the amount of time that many youths with psychosis spent at the hospital emergency.

$20654 \%$ of emergency department referrals had their intake assessment take place in ambulatory settings 207 such as the emergency room, outpatient settings (including at PEPP itself), or in the community. The 
208 remainder required hospitalization. Thus, the frontline deployment of the intake clinician may have 209 averted inpatient hospitalization for over half the youths presenting with psychosis at one psychiatric

210 emergency unit. It is also likely to have reduced the referral delay for patients referred from the ER

211 (median: 0.42 weeks, range -8.57 to 196 weeks) that would have occurred if emergency staff simply 212 discharged clients with PEPP's contact information. These are prominent illustrations of how PEPP's

213 intake clinician gives young people a "soft landing" into early intervention services by bypassing

214 potentially traumatic pathways to care.

215 Over time, PEPP's intake clinician has fostered relationships with numerous catchment-area healthcare 216 and community organizations. A semi-structured interview, called Circumstances of Onset and Relapse

217 Schedule(Norman and Malla, 2002) has recorded every client's sources of referral, help-seeking contacts 218 before entering PEPP and treatment delay indices. This interview, conducted by trained research staff 219 within the first few months following entry to PEPP, constructs a timeline of the client's life, from the 220 development of symptoms to their help-seeking efforts until entry to PEPP. The timeline is presented to 221 the research team, including one senior psychiatrist, and consensus is reached on several key measures 222 such as DUP, defined as the time between onset of psychotic symptoms and commencement of one 223 month of continuous antipsychotic treatment. In addition to furthering research (Bechard-Evans et al., 224 2007, Cassidy et al., 2008, Malla et al., 2005, Malla et al., 2014), these indices continuously inform 225 outreach targets and activities.

227 At PEPP, the median DUP for all accepted clients is 114 days/16.35 weeks (range: $0-512$ days). This is 228 significantly shorter than the DUPs of 6-12 months, or more, that many other early intervention services 229 report (Albert et al. 2017, Lloyd-Evans et al., 2011, Marshall et al., 2005). PEPP's median referral delay is 2301.28 weeks (range: -13.2- 445 weeks). This short referral delay may at least in part result from the intake 231 clinician's strong links with community referral sources. A negative value indicates that a client was 232 referred to PEPP before developing full-threshold symptoms, for example, if the client was experiencing 233 sub-threshold psychotic symptoms. Specifically, these clients were first followed in PEPP's sub-program 234 for youth at ultra-high risk for psychosis (Pruessner et al., 2015) and immediately accepted into the PEPP 
235 program when they developed threshold-level psychotic episode. In these cases, their initial referral date 236 to PEPP is used, hence the negative measure of delay.

\section{Discussion}

238 PEPP's rapid access system and its demonstrated impacts in reducing wait-times to services are 239 particularly noteworthy in Canada, where long wait lists and delays before services can be accessed are 240 the norm (Barua et al. 2015, Kowaleski et al. 2011).

241 One of the chief components contributing to rapid access to our program is an open referral system, 242 which bypasses the more traditional filter system where primary care is a required first step to accessing 243 psychiatric services (Goldberg and Huxley, 1990). However, even given the possibility that youth or 244 informal sources of help could contact PEPP directly, the majority of referrals still arose from formal health 245 services. This reflects the need to not only reduce systemic barriers to services, but also to carry out 246 active community outreach to increase self- and carer referrals.

247 The increase in diversity in referral sources to PEPP over the past 14 years may represent an increase in 248 awareness of the clinic, following media coverage, and/or an increase in awareness of the signs and 249 symptoms of psychosis, especially among community-based primary care settings and the educational 250 sector. Both these areas were targeted through formalized early identification initiatives directly aimed at 251 potential referral sources in the community.

253 The intake and initial evaluation model described herein has been implemented effectively since 1997 at 254 the PEPP program in London, Ontario and since 2003 at PEPP-Montreal. Its replicability is attested to by 255 its deployment at three other first-episode psychosis programs in Montreal. These deployments were 256 done in 2008, 2010 and 2012 facilitated by PEPP-Montreal's training and knowledge translation activities.

257 Though these other Montreal early intervention services have adopted a similar model for rapid intake, 258 none have evaluated its effectiveness through a systematic assessment of DUP and specifically, of 259 referral and engagement delays. Thus, we cannot confidently comment on whether the same success in 260 reducing delays has been achieved by these similar programs.

261 This is important to note, given that 'timely contact with referred individuals' was highlighted by expert 
262 consensus as one of the chief components of early intervention for psychosis services (Addington, et al.

263 2013). Yet in many cases, early intervention services either do not reach this target or do not have the

264 available data to report on this component (White et al. 2015).

266 Our model requires a commitment to ensuring rapid access that goes beyond a philosophical framework

267 and necessitates additional resources, including well-established clinical supervision. Daily challenges

268 described by PEPP intake clinicians include occasionally dealing with high referral volumes; engaging

269 clients who are reluctant to enter services; and matching individuals who do not meet our intake criteria to

270 other more appropriate services. Many of these other services do not share a similar emphasis on rapid

271 access and have long waiting lists.

272

273 Concluding thoughts

274 Despite wide acceptance of the need for rapid-access early intervention services, their availability and

275 accessibility remain inconsistent. Our protocol's demonstrated success in providing rapid, engaging

276 access to high-quality first-episode psychosis services makes it a model for early intervention services for 277 psychosis and other mental illnesses.

\section{$278 \quad$ References}

279 Addington DE, McKenzie E, Norman R, Wang J, Bond GR. Essential evidence-based components of first280 episode psychosis services. Psychiatric Services. 2013 May;64(5):452-7.

281 Albert, Nikolai, Marianne Melau, Heidi Jensen, Charlotte Emborg, Jens Richardt Mollegaard Jepsen, 282 Birgitte Fagerlund, Christian Gluud, Ole Mors, Carsten Hjorthøj, and Merete Nordentoft. "Five 283 years of specialised early intervention versus two years of specialised early intervention followed 284 by three years of standard treatment for patients with a first episode psychosis: randomised, 285 superiority, parallel group trial in Denmark (OPUS II)." BMJ. 356 (2017): i6681. 384-395. 
290 Andrade, L., Alonso, J., Mneimneh, Z., Wells, J., Al-Hamzawi, A., Borges, G., Bromet, E., Bruffaerts, R., 291 De Girolamo, G. \& De Graaf, R. 2014. Barriers To Mental Health Treatment: Results From The 292 Who World Mental Health Surveys. Psychological Medicine, 44, 1303-1317.

293 Barua, Bacchus, Mark Rovere, and Brett J. Skinner. Waiting your turn. Fraser Institute, 2015.

294 Bechard-Evans, L., Schmitz, N., Abadi, S., Joober, R., King, S. \& Malla, A. 2007. Determinants Of Help-

295 Seeking And System Related Components Of Delay In The Treatment Of First-Episode 296 Psychosis. Schizophrenia Research, 96, 206-214.

297 Birchwood, M. 1999. Early Intervention In Psychosis: The Critical Period. In: Mcgorry, P. D. \& Jackson, H.

298 J. (Eds.) The Recognition And Management Of Early Psychosis: A Preventive Approach.

299 Cambridge, Uk: Cambridge University Press.

300 Boydell, K. M., Gladstone, B. M. \& Volpe, T. 2006. Understanding Help Seeking Delay In The Prodrome 301 To First Episode Psychosis: A Secondary Analysis Of The Perspectives Of Young People. 302 Psychiatric Rehabilitation Journal, 30, 54-60.

303 Cassidy, C. M., Schmitz, N., Norman, R., Manchanda, R. \& Malla, A. 2008. Long-Term Effects Of A 304 Community Intervention For Early Identification Of First-Episode Psychosis. Acta Psychiatrica 305 Scandinavica, 117, 440-448.

306 Coldham, E. L., Addington, J. \& Addington, D. 2002. Medication Adherence Of Individuals With A First 307 Episode Of Psychosis. Acta Psychiatrica Scandinavica, 106, 286-290.

308 Doyle, R., Turner, N., Fanning, F., Brennan, D., Renwick, L., Lawlor, E. \& Clarke, M. 2014. First-Episode 309 Psychosis And Disengagement From Treatment: A Systematic Review. Psychiatric Services. 310 Friis, S., Vaglum, P., Haahr, U., Johannessen, J. O., Larsen, T. K., Melle, I., Opjordsmoen, S., Rund, B. 311 R., Simonsen, E. \& Mcglashan, T. H. 2005. Effect Of An Early Detection Programme On Duration 312 Of Untreated Psychosis: Part Of The Scandinavian Tips Study. Br J Psychiatry Suppl, 48, S29$313 \quad 32$.

314 Huxley, Peter. "Mental illness in the community: the Goldberg-Huxley model of the pathway to psychiatric 315 care." Nordic Journal of Psychiatry 50, no. sup37 (1996): 47-53.

316 International Early Psychosis Association Writing Group. 2005 International Clinical Practice Guidelines 317 For Early Psychosis. The British Journal Of Psychiatry, 187, S120-S12. 
318 Iyer, S., Boksa, P., Lal, S., Shah, J., Marandola, G., Jordan, G., Doyle, M., Joober, R. \& Malla, A. $2015 a$. Transforming Youth Mental Health: A Canadian Perspective. Irish Journal Of Psychological Medicine, 32, 51-60.

321 Iyer, S., Jordan, G., Macdonald, K., Joober, R. \& Malla, A. 2015b. Early Intervention For Psychosis: A 322 Canadian Perspective. J Nerv Ment Dis, 203, 356-64.

323 Iyer, S. N. M., A 2014. Early Intervention In Psychosis: Concepts, Current Knowledge And Future 324 Directions. Santé Mentale Au Québec 39.

325 Kowalewski, Karolina, John D. McLennan, and Patrick J. McGrath.A preliminary investigation of wait 326 times for child and adolescent mental health services in Canada Journal of the Canadian 327 Academy of Child \& Adolescent Psychiatry 20, no. 2 (2011).

328 Lloyd-Evans, B., Crosby, M., Stockton, S., Pilling, S., Hobbs, L., Hinton, M. \& Johnson, S. 2011. Initiatives 329 To Shorten Duration Of Untreated Psychosis: Systematic Review. The British Journal Of $330 \quad$ Psychiatry, 198, 256-263.

331 Malla, A., Iyer, S., Mcgorry, P., Cannon, M., Coughlan, H., Singh, S., Jones, P. \& Joober, R. 2015. From 332 Early Intervention In Psychosis To Youth Mental Health Reform: A Review Of The Evolution And 333 Transformation Of Mental Health Services For Young People. Social Psychiatry And Psychiatric $334 \quad$ Epidemiology, 51, 1-8.

335 Malla, A., Jordan, G., Joober, R., Schmitz, N., Norman, R., Brown, T., Goldberg, K., Loohuis, H., 336 Vracotas, N. \& Rochford, J. 2014. A Controlled Evaluation Of A Targeted Early Case Detection 337 Intervention For Reducing Delay In Treatment Of First Episode Psychosis. Social Psychiatry And $338 \quad$ Psychiatric Epidemiology, (Accepted With Revisions).

339 Malla, A., Norman, R., Scholten, D., Manchanda, R. \& Mclean, T. 2005. A Community Intervention For 340 Early Identification Of First Episode Psychosis. Social Psychiatry And Psychiatric Epidemiology, $341 \quad 40,337-344$.

342 Marshall, M., Lewis, S., Lockwood, A., R, D., Jones, P. \& Croudace, T. 2005. Association Between 343 Duration Of Untreated Psychosis And Outcome In Cohorts Of First-Episode Patients. Arch Gen $344 \quad$ Psychiatry, 62, 975-983. 
345 Mcgorry, P. D., Edwards, J., Mihalopoulos, C., Harrigan, S. M. \& Jackson, H. J. 1996. Eppic: An Evolving System Of Early Detection And Optimal Management. Schizophrenia Bulletin, 22, 305.

347 Nolin, M., Malla, A., Tibbo, P., Norman, R. \& Abdel-Baki, A. 2016. Early Intervention For Psychosis In 348 Canada What Is The State Of Affairs? The Canadian Journal Of Psychiatry, 61, 186-194.

349 Norman, R. \& Malla, A. 2002. Course Of Onset And Relapse Schedule: Interview And Coding Instruction $350 \quad$ Guide. Prevention And Early Intervention For Psychosis Program, London, Ontario, Canada.

351 Norman, R., Malla, A., Verdi, M., Hassall, L. \& Fazekas, C. 2004. Understanding Delay In Treatment For $352 \quad$ First-Episode Psychosis. Psychological Medicine, 34, 255-266.

353 Norman, R. M. \& Malla, A. K. 2001. Duration Of Untreated Psychosis: A Critical Examination Of The $354 \quad$ Concept And Its Importance. Psychological Medicine, 31, 381-400.

355 Pruessner, M., Faridi, K., Shah, J., Rabinovitch, M., Iyer, S., Abadi, S., Pawliuk, N., Joober, R. \& Malla, A. 356 K. 2015. The Clinic For Assessment Of Youth At Risk (Cayr): 10 Years Of Service Delivery And 357 Research Targeting The Prevention Of Psychosis In Montreal, Canada. Early Intervention In $358 \quad$ Psychiatry.

359 Rickwood, D. J., Deane, F. P. \& Wilson, C. J. 2007. When And How Do Young People Seek Professional $360 \quad$ Help For Mental Health Problems? Medical Journal Of Australia, 187, S35.

361 Shah, J. 2015. Sub-Threshold Mental Illness In Adolescents: Within And Beyond Dsm's Boundaries.

363 Singh, S. P. \& Grange, T. 2006. Measuring Pathways To Care In First-Episode Psychosis: A Systematic 364 Review. Schizophrenia Research, 81, 75-82.

365 Srihari, V. H., Tek, C., Pollard, J., Zimmet, S., Keat, J., Cahill, J. D., Kucukgoncu, S., Walsh, B. C., Li, F. 366 \& Gueorguieva, R. 2014. Reducing The Duration Of Untreated Psychosis And Its Impact In The 367 Us: The Step-Ed Study. Bmc Psychiatry, 14, 1.

368 Szmukler, G. I. \& Bloch, S. 1997. Family Involvement In The Care Of People With Psychosis. British $369 \quad$ Journal Of Psychiatry, 171, 401-405.

370 White, Dominique A., Lauren Luther, Kelsey A. Bonfils, and Michelle P. Salyers. "Essential components 371 of early intervention programs for psychosis: Available intervention services in the United 372 States." Schizophrenia research168, no. 1 (2015): 79-83. 
2

3

4

5

6

7

8

9

10

11

12

13

14

15

16

17

18

19

20

21

22

23

24

25

26

27

28

29

30

31

32

33

34

35

36

37

38

39

40

41

42

43

44

45

46

47

48

49

50

51

52

53

54

55

56

57

58

59

60

\section{$373 \quad$ Figure Legends}

374 Figure 1. Referral sources to PEPP over the years from 2003-2015

375 Figure 2. Referral and intake procedure at PEPP

376

377

378

379

380 
Figure 1. Referral sources to PEPP over the years from 2003-2015.

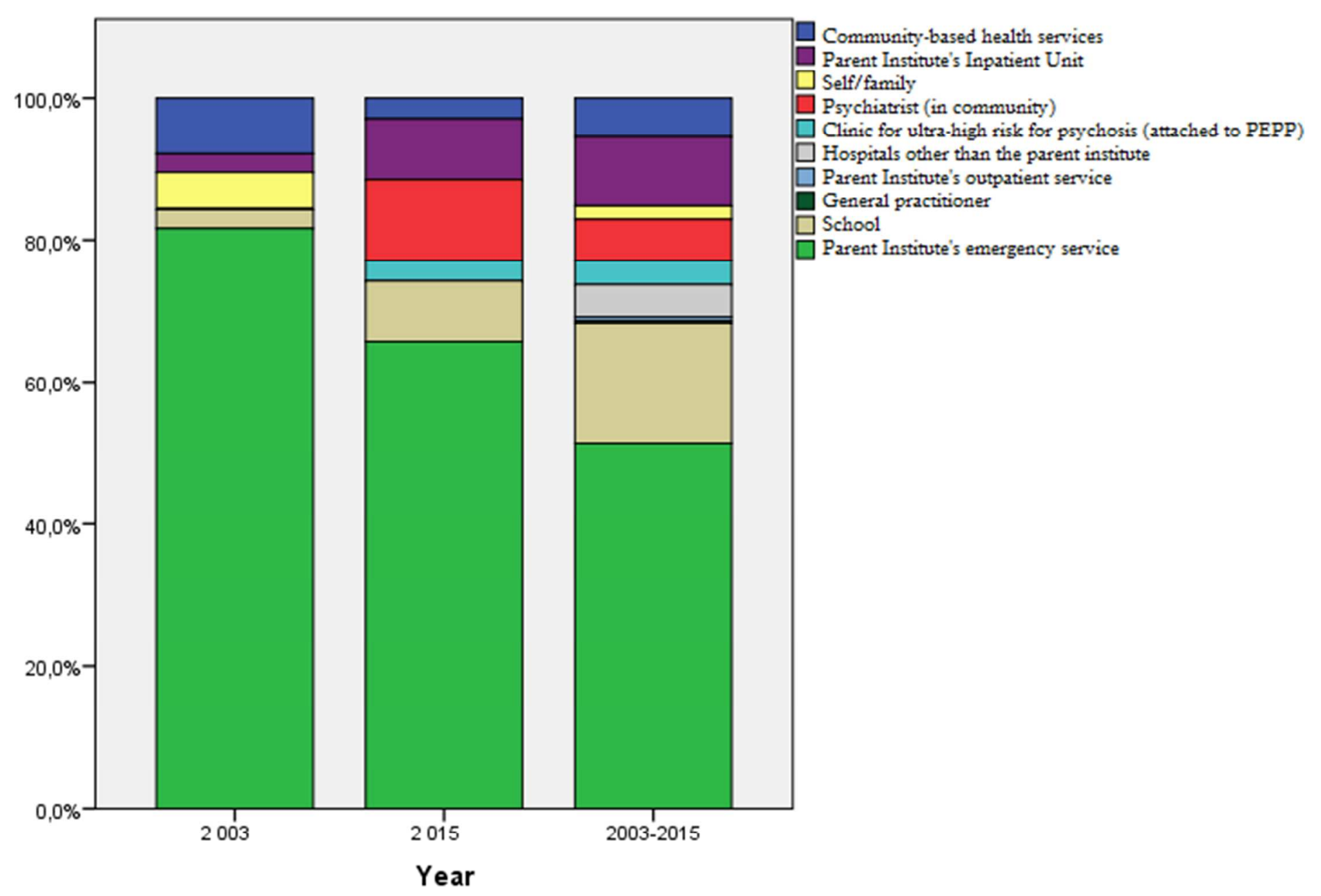

PEPP is a progam located in a larger psychiatric institution called the Douglas Mental Health University Institute which houses other services like the ER, non-first episode psychosis outpatient services, inpatient services, etc. 


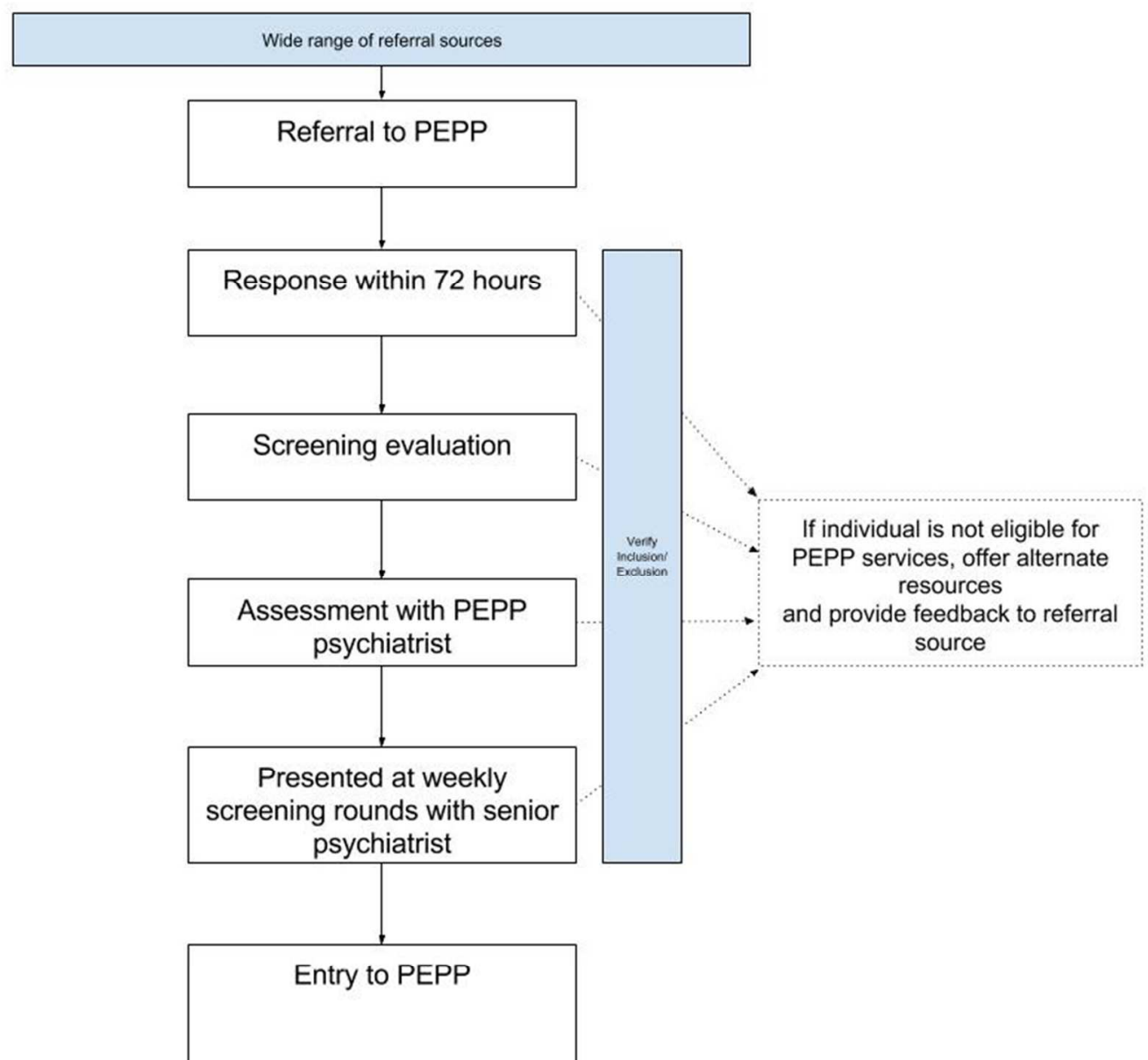

Figure 3. Referral and intake procedure at PEPP 